\title{
ASYMPTOTIC INVERSION OF LAPLACE TRANSFORMS: A CLASS OF COUNTEREXAMPLES
}

\author{
JOHN S. LEW
}

\begin{abstract}
Let $f$ be a complex-valued locally integrable function on $[0,+\infty)$, and let $L f$ be its Laplace transform, whenever and wherever it exists. We review some known methods, exact and approximate, for recovering $f$ from $L f$. Since numerical algorithms need auxiliary information about $f$ near $+\infty$, we note that the behavior of $f$ near $+\infty$ depends on the behavior of $L f$ near $0+$, hence that our ability to retrieve $f$ is limited by the class of momentless functions, namely, all functions $f$ such that $L f(s)$ converges absolutely for $\operatorname{Re}(s)>0$ and satisfies

$$
L f(s)=o\left(s^{n}\right) \text { near } 0+\quad \text { for } n=0,1,2, \cdots .
$$

We discuss the space $Z$ of momentless functions and complex distributions, then construct a family of elements in this space which defy various plausible conjectures.
\end{abstract}

1. Introduction. Let $f$ be a complex-valued locally integrable function on $[0,+\infty)$, and let

$$
L[f ; s]=\int_{0}^{\infty} \exp (-s t) f(t) d t
$$

be its Laplace transform, whenever and wherever this integral exists. Indeed [21, pp. 96-102] for some $\sigma_{a}(f)$ and $\sigma_{c}(f)$ with $-\infty \leqq \sigma_{c}(f) \leqq$ $\sigma_{a}(f) \leqq+\infty$ there are maximal half-planes $\operatorname{Re}(s)>\sigma_{a}(f)$ and $\operatorname{Re}(s)>\sigma_{c}(f)$ in which respectively $L[f ; s]$ is absolutely convergent and conditionally convergent. If these half-planes are nonvoid, then $L[f, s]$ is also holomorphic at least in $\operatorname{Re}(s)>\sigma_{c}(f)$, and $f(t)$ is uniquely determined by $L[f ; s]$ except on a set of measure zero [21, pp. 99, 108].

An important step in many problems is the inversion of a Laplace transform, that is, the recovery of $f(t)$ from $L[f ; s]$. Sometimes this can be accomplished exactly through transform tables (e.g. [5]), inversion formulas $([3$, p. 286], [6], [21, pp. 108, 141]), or convergent series ([3, pp. 301-305], [17, p. 97], [18], [19, Chapter 9]). However many inversions

Received by the editors September 22, 1972.

AMS (MOS) subject classifications (1970). Primary 41A60, 44A10; Secondary 30A84, 44A40.

Key words and phrases. Laplace transform, asymptotic inversion, inverse Abelian theorem, Tauberian theorem, Mellin series.

(c) American Mathematical Society 1973 
employ numerical techniques ([1], [2]), which typically lose accuracy for large $t$; hence such algorithms require further information which describes $f(t)$ approximately near $+\infty$. Theorems which derive the limiting behavior of $f(t)$ from that of $L[f ; s]$ are called respectively Tauberian or inverse Abelian according as they involve extra hypotheses on $f(t)$ or on $L[f ; s]$.

If $f(t)$ can be expressed by the inversion integral [21, p. 108]

$$
f(t)=(2 \pi i)^{-1} \int_{c-i \infty}^{c+i \infty} \exp (t s) L[f ; s] d s,
$$

if $L[f ; s]$ can be continued analytically to the left, and if the contour of (1.2) can be moved sufficiently in that direction, then the behavior of $f(t)$ near $+\infty$ is determined by that of $L[f ; s]$ near its rightmost singularities. If these singular points are all poles then their contributions are simple residues and $[4$, p. 110]

$$
f(t) \sim \sum_{m=0}^{\infty} P_{m}(t) \exp [a(m) t] \text { near }+\infty,
$$

with $P_{m}$ a polynomial for each $m$, and $\operatorname{Re}[a(m)] \downarrow-\infty$ as $m \rightarrow \infty$. An essential singularity of $L[f ; s]$ yields a Taylor expansion for $\boldsymbol{P}_{m}[3, \mathrm{p} .488]$.

If $L[f ; s]$ has a branch point $s_{0}$ as its unique rightmost singularity, then $s_{0}$ may be shifted to the origin without loss of generality. Thus for any positive $a$ we can infer $\int_{0}^{t} f(u) d u \sim c t^{a} / \Gamma(1+a)$ near $+\infty$ by the Tauberian theorem of Karamata [19, p. 197], given that $L[f ; s] \sim c s^{-a}$ near $0+$ and that $f(t)+k t^{a-1} \geqq 0$ for some $k$. Also for any complex $a$ we can infer that $f(t) \sim c t^{a-1} / \Gamma(a)$ near $+\infty$ by inverse Abelian theorems of Doetsch, given that $L[f ; s] \sim c s^{-a}$ near 0 in a sector $|\arg s| \leqq \theta$, where either $\theta>\pi / 2$ or $\theta=\pi / 2$, and in the latter case $L[f ; s]$ satisfies further conditions on the imaginary axis. For generalized functions some results of this kind have been proved by Lavoine [12], involving the regularized functions $c t^{a-1}$; for $\log f(t)$ some estimates near $+\infty$ have been obtained by Wagner ([17], [18], [25]), describing still more singular behavior.

To get sharper results at a branch point, we consider series

$$
f(t) \sim \sum_{m=0}^{\infty} P_{m}(\log t) t^{a(m)} \quad \text { near }+\infty
$$

with $P_{m}$ and $\operatorname{Re}[a(m)]$ as in (1.3). Expansions near $+\infty$ of this form, or near $0+$ with $\operatorname{Re}[a(m)] \uparrow+\infty$, were originally treated, it seems, by Mellin [13], and are thus called Mellin series or expansions by the author. If the function $f$ has a Mellin series near $+\infty$ then this series for $f$, and certain values of its Mellin transform, determine systematically a Mellin series 
for $L[f ; s]$ near $0+[7]$; while this series for $L[f ; s]$ and the existence of an expansion (1.4) determine uniquely the Mellin series for $f(t)$ near $+\infty$ ([8], [9]). The existence and form of a series (1.4) follows by two inverse Abelian theorems of Doetsch ([4, pp. 150-160], [9], [11]) from assumptions on $L[f ; s]$ in a sector $|\arg s| \leqq \theta$, where either $\theta>\pi / 2$ or $\theta=\pi / 2$, and in the latter case $L[f ; s]$ satisfies further conditions on the imaginary axis. However these assumptions on $L[f, s]$ are not necessary [9, Example 4]. These results of Doetsch have also been extended in work of Riekstina ([23], [24]).

To explore all possibilities for theorems of this kind, we remark that if $g$ is rapidly decreasing near $+\infty$ then $L[g ; s]$ can be expanded by moments:

$$
L[g ; s] \sim \sum_{n=0}^{\infty} \mu_{n}(-s)^{n} / n ! \quad \text { near } 0+\quad \text { with } \mu_{n}=\int_{0}^{\infty} t^{n} g(t) d t .
$$

Thus any transformable $g$ will be called a momentless function if $\sigma_{a}(g) \leqq 0$ and

$$
L[g ; s]=o\left(s^{n}\right) \quad \text { near } 0+\quad \text { for all } n=0,1,2, \cdots .
$$

A nontrivial example from standard tables $[5$, p. 158] is

$$
\begin{aligned}
g(t) & =t^{-1 / 2} \cos (k t)^{1 / 2} \quad \text { with } k>0, \\
L[g ; s] & =(\pi / s)^{1 / 2} \exp (-k / 4 s) .
\end{aligned}
$$

If $g$ is a function of this kind then $L[f ; s]$ and $L[f+g ; s]$ have identical Mellin series near $0+$. Thus the Mellin series for $f$ is not recoverable unless all permissible $g$ are rapidly decreasing under the set of hypotheses for a conjectured theorem. We shall therefore construct a class of momentless functions and distributions through which we may eliminate a number of conjectures on asymptotic inversion.

2. Notation. We shall construct the desired counterexamples on $[0,+\infty)$ as a family of functions and measures, but can introduce the associated concepts more easily in a space of generalized functions. Indeed if $D_{+}^{\prime}$ is the space of Schwartz distributions on $(-\infty,+\infty)$ with support in $[0,+\infty)$ then $D_{+}^{\prime}$ is a commutative algebra over the complex field under "pointwise" addition, scalar multiplication, and the standard convolution ([15, pp. 113, 121], [22, pp. 122-130]). This convolution $f * g$ for elements of $D_{+}^{\prime}$ extends the definition

$$
[f * g](t)=\int_{0}^{t} f(t-u) g(u) d u
$$

for functions on $[0,+\infty)[15$, p. 115]. 
Within $D_{+}^{\prime}$ let $e$ represent the Dirac delta "function", so that $e$ is the identity for this algebra, and let $1_{+}$denote the Heaviside step function, so that

$$
\left[1_{+} * f\right](t)=\int_{0}^{t} f(u) d u
$$

for functions on $[0,+\infty)$. Then we can define

$$
f^{* 0}=e, \quad f^{* 1}=f, \quad f^{* n+1}=f * f^{* n}
$$

for any $f$ in $D_{+}^{\prime}$ and all $n=1,2, \cdots$. Moreover $D_{+}^{\prime}$ is closed under

$$
f \rightarrow 1_{+} * f, \quad f \rightarrow d f / d t,
$$

and the first of these mappings is the inverse of the second.

For any element $f$ of $D_{+}^{\prime}$ and any complex $s=c+i u$ the Laplace transform $L[f ; s]$ is defined $([15$, p. 217], [22, p. 222]) as the Fourier transform

$$
\int_{0}^{\infty} \exp (-i t u) \exp (-c t) f(t) d t
$$

whenever $\exp (-c t) f(t)$ is in the space $S^{\prime}$, so that $(2.5)$ is a well-defined entity. Then for some value $\sigma(f)$, either real or $\pm \infty$, the transform $L[f ; s]$ is defined and analytic on $\operatorname{Re}(s)>\sigma(f)$, and for functions on $[0,+\infty)$ this half plane of existence includes the preceding $\operatorname{Re}(s)>\sigma_{a}(f)$ ([15, p. 218], [22, p. 223]).

Now consider the set $A$ of all $f$ in $D_{+}^{\prime}$ such that $L[f ; s]$ is defined in this sense for $\operatorname{Re}(s)>0$ at least and such that

$$
L[f ; s]=O\left(s^{k}\right) \text { for some real } k
$$

as $s \rightarrow 0$ in this half plane. Clearly $A$ is a subalgebra of $D_{+}^{\prime}$ by the convolution theorem $([15$, p. 222], [22, p. 240]), and is closed under the mappings (2.4) by the identities ([15, pp. 222-223], [22, p. 228])

$$
L\left[1_{+} * f ; s\right]=s^{-1} L[f ; s], \quad L[d f \mid d t ; s]=s L[f ; s] .
$$

Call $f$ momentless if it lies in $A$ and satisfies (1.6); define $Z$ as the set of all such $f$. Then $Z$ is an ideal in $A$ by (1.6) and (2.6), while $Z$ is closed under (2.4) by (1.6) and (2.7).

Within $A$ denote by $J$ the space of all elements $f$ which correspond to locally integrable functions, modulo the space of all functions which vanish except on null sets. The introduction of $J$ offers a criterion for $A$ : if $f$ is given in $D_{+}^{\prime}$ then $f$ is also in $A$ whenever $1_{+}^{* n} * f$ is in $J$ for some $n=0,1,2, \cdots$, and

$$
\left[1_{+}^{* n} * f\right](t)=o\left(t^{k}\right) \text { near }+\infty
$$


for some $k>0$. Indeed, under these conditions $L\left[1_{+}^{* n} * f ; s\right]$ is absolutely convergent on $\operatorname{Re}(s)>0$ and is $o\left(s^{-k-1}\right)$ as $s \rightarrow 0$ ([19, p. 182], [22, p. 249]); so that $L[f ; s]$ satisfies $(2.6)$ by use of (2.7). However this criterion is not necessary, for

$$
g(t)=\sum_{n=0}^{\infty}(d / d t)^{n} e(t-n)
$$

is in $A$, but no $1_{+}^{* n} * g$ is in $J$.

Let $M$ be the set of all $f$ in $A$ which correspond to complex measures on $[0,+\infty)$, namely, those for which $1_{+} * f$ has locally bounded variation. Then $M$ is a subalgebra of $A[19, \mathrm{p} .84]$; measure algebras are discussed in standard works ([10, pp. 141-150], [14, pp. 13-17]). Let $C^{n}$ be the set of all $f$ in $J$ which have $n$ continuous derivatives on $(-\infty,+\infty)$, for all $n=0,1,2, \cdots$ or $\infty$. Then $J$ is an ideal in $M\left[10\right.$, p. 143], all $C^{n}$ are ideals in $M[15, \mathrm{p} .122]$, and $C^{\infty}$ is closed under (2.4).

Finally, we collect these remarks on algebraic structure to obtain the following ideals in the system $M$ :

$$
Z \cap M, Z \cap J, \quad Z \cap C^{n} \text { for } n=0,1, \cdots, \infty .
$$

Therefore we can generate elements of $Z \cap M$ with arbitrary preassigned smoothness from a special family $\left\{h_{a, x}\right\}$ with $a$ and $x$ suitable real numbers. Also we can construct more singular elements of $Z$ by repeated differentiation of $h_{a, x}$.

3. Construction. For any real $a$ and $x$ the expression

$$
K(a, x, z)=(1-z)^{-1-a} \exp [x z /(z-1)]
$$

is analytic in the complex $z$ plane cut from 1 to $+\infty$, and is the generating function [16, equation (5.1.9)] for the generalized Laguerre polynomials $L_{n}^{(a)}(x)$, so that

$$
K(a, x, z)=\sum_{n=0}^{\infty} L_{n}^{(a)}(x) z^{n} \quad \text { for } \quad|z|<1 .
$$

Moreover, by Fejer's formula [16, equation (8.22.1)],

$$
\begin{aligned}
L_{n}^{(a)}(x)= & \pi^{-1} \exp (x / 2) x^{(-2 a-1) / 4} \cdot n^{(2 a-1) / 4} \cdot \cos \left[2(n x)^{1 / 2}-(2 a+1) \pi / 4\right] \\
& +O\left[n^{(2 a-3) / 4}\right] \text { as } n \rightarrow+\infty
\end{aligned}
$$

uniformly on any compact interval in $0<x<+\infty$.

On $|z| \leqq 1$ the series (3.2) converges absolutely for $a<-\frac{3}{2}$ by the last formula, and conditionally for $a=-\frac{3}{2}$ by Littlewood's theorem [21, p. 215]. However for each positive $x$ the set

$$
\left\{2(n x)^{1 / 2}-(2 a+1) \pi / 4: n=0,1,2, \cdots\right\} \text { modulo } 2 \pi
$$


is dense in $[0,2 \pi)$, so that, with any positive $\delta$ and $n_{0}$,

$$
\left|L_{n}^{(a)}(x)\right| \geqq(1-\delta) \pi^{-1} \exp (x / 2) \cdot x^{(-2 a-1) / 4} \cdot n^{(2 a-1) / 4}
$$

for some $n>n_{0}$. Thus $L_{n}^{(a)}(x)$ for large $n$ is not $O\left(n^{r}\right)$ unless $r \geqq(2 a-1) / 4$.

For any fixed real $a$ and positive $x$, letting $e(t)$ be the Dirac delta function, we construct

$$
h_{a, x}(t)=\sum_{n=0}^{\infty} L_{n}^{(a)}(x) e(t-n) .
$$

By Abel's theorem [21, pp. 27-28] if $a \leqq-\frac{3}{2}$ then

$$
\left[1_{+} * h_{a, x}\right](+\infty)=\sum_{n=0}^{\infty} L_{n}^{(a)}(x)=\lim _{z \rightarrow 1-} K(a, x, z)=0 .
$$

By this relation and (3.3), if $a$ is arbitrary then

$$
\left[1_{+} * h_{a, x}\right](t)=O\left[t^{(2 a+3) / 4}\right] \text { as } t \rightarrow+\infty .
$$

Hence by (2.8) these $h_{a, x}$ are elements of $M$ with support on the nonnegative integers. Moreover these $h_{a, x}$ are elements of $Z$, since if $\operatorname{Re}(s)>0$ then

$$
\begin{aligned}
L\left[h_{a, x} ; s\right] & =\sum_{n=0}^{\infty} L_{n}^{(a)}(x) \exp (-n s)=K(a, x, \exp (-s)) \\
& =o\left(s^{m}\right) \text { near } 0+\quad \text { for } m=0,1,2, \cdots .
\end{aligned}
$$

EXAMPLE 1. Before finding this construction the author advanced the conjecture that if $f$ were an element of $Z$ which was bounded as a measure on $[0,+\infty)$ then

$$
\left[1_{+} * f\right](+\infty)-\left[1_{+} * f\right](t)=o\left(t^{-n}\right) \text { near }+\infty \quad \text { for all } n>0 \text {. }
$$

However if $a<-\frac{3}{2}$ then $h_{a, x}$ is a bounded measure on [0, $+\infty$ ) by (3.3), and $h_{a, x}$ is algebraically decaying near $+\infty$ by (3.5). Indeed some multiple of $h_{a, x}$, by (3.7), is the difference of two probability measures on the integers; hence these measures differ by a momentless distribution which decays no faster than $t^{(2 a-1) / 4}$.

EXAMPLE 2. One might suppose that matters would improve for functions $f$ in $Z \cap L^{1}(-\infty,+\infty)$. However let $f=g * h_{a, x}$, where $g$ is intuitively any function in $L^{1}[0,+\infty)$, or technically any function in $J \cap L^{1}(-\infty,+\infty)$. Then by (2.10), $f$ is in $Z \cap J$ for all real $a$, and by (3.3), $f$ is in $Z \cap L^{1}(-\infty,+\infty)$ for $a<-\frac{3}{2}$. Moreover if $g$ is unbounded at the origin and is bounded outside each neighborhood of zero, then $f$ or some multiple is unbounded at $t=0,1,2, \cdots$ and is the difference between two probability densities.

EXAMPLE 3. One might expect more from the intersection of $Z$ with some Sobolev space. However if $g$ is $C^{\infty}$ with support in $(0,1)$ and if 
$f=g * h_{a, x}$ with $h_{a, x}$ as defined, then $f$ is in $Z \cap C^{\infty}$ by (2.10) and $f$ is in $L^{p}(-\infty,+\infty)$ by (3.3) whenever

$$
\sum_{n=1}^{\infty} n^{(2 a-1) p / 4}<+\infty,
$$

hence for all $p$ in $[1,+\infty]$ whenever $a<-\frac{3}{2}$. Moreover $f^{(m)}=g^{(m)} * h_{a, x}$, so that $f^{(m)}$ has the same properties as $f$, and thus $f^{(m)}$ is in $L^{p}(-\infty,+\infty)$ for all $p$ in $[1,+\infty]$ and all $m=0,1,2, \cdots$. At the same time $f$ and its derivatives decay no faster than $t^{(2 a-1) / 4}$.

These examples show that conditions on $f$ of smoothness and integrability cannot produce estimates of $f(t)$ near $+\infty$ from estimates of $L[f ; s]$ near $0+$. Indeed for any fixed $a$ and $x$, the numbers

$$
\left\{L_{n}^{(a)}(x): n=0,1,2, \cdots\right\}
$$

change sign according to (3.3), so that the functions $f=g * h_{a, x}$ oscillate systematically as $t \rightarrow+\infty$. Thus the positivity condition of Karamata's theorem serves to exclude some elements of $Z$. However this theorem applies only to functions $f$ for which $1_{+} * f$ grows algebraically near $+\infty$, whence new results might well arise from other hypotheses under which $f$ oscillates negligibly near $+\infty$.

\section{REFERENCES}

1. R. E. Bellman, R. E. Kalaba and J. A. Lockett, Numerical inversion of the Laplace transform: Applications to biology, economics, engineering and physics, American Elsevier, New York, 1966. MR 34 \#5282.

2. J. W. Cooley, P. A. W. Lewis and P. D. Welch, The fast Fourier transform algorithm: programming considerations in the calculation of sine, cosine, and Laplace transforms, J. Sound Vib. 12 (1970), 315-337.

3. G. Doetsch, Handbuch der Laplace-Transformation. Band I: Theorie der LaplaceTransformation, Verlag Birkhäuser, Basel, 1950. MR 13, 230.

4. - Handbuch der Laplace-Transformation. Band II: Andwendungen der Laplace-Transformation, Verlag Birkhäuser, Basel, 1955. MR 18, 35.

5. A. Erdélyi et al., Tables of integral transforms. Vol. I, McGraw-Hill, New York, 1954. MR 15, 868.

6. H. Goldenberg, The evaluation of inverse Laplace transforms without the aid of contour integration, SIAM Rev. 4 (1962) 94-104. MR 25 \#397.

7. R. A. Handelsman and J. S. Lew, Asymptotic expansion of Laplace transforms near the origin, SIAM J. Math. Anal. 1 (1970), 118-130. MR 41 \#4142.

8. - Asymptotic expansion of Laplace convolutions for large argument, SIAM Rev. 13 (1971), 269.

9. - Asymptotic expansion of Laplace convolutions for large argument and tail densities for certain sums of random variables, SIAM J. Math. Anal. (to appear).

10. E. Hille and R. S. Phillips, Functional analysis and semi-groups, rev. ed., Amer. Math. Soc. Colloq. Publ., vol. 31, Amer. Math. Soc., Providence, R.I., 1957. MR 19, 664. 
11. T. E. Hull and C. Froese, Asymptotic behavior of the inverse of a Laplace transform, Canad. J. Math. 7 (1955), 116-125. MR 16, 584.

12. J. Lavoine, Sur les théorèmes abéliens et taubériens de la transformation de Laplace, Ann. Inst. Henri Poincaré 4 (1966), 49-65. MR 34 \#6452.

13. H. Mellin, Abriss einer allgemeinen und einheitlichen Theorie der asymptotische Reihen, Wissenschaftliche Vorträge gehalten auf dem 5 Kongress der Skandinav. Mathematiker in Helsingfors, 4-7 Juli 1922, vol. 1, 1922, pp. 1-17.

14. W. Rudin, Fourier analysis on groups, Interscience Tracts in Pure and Appl. Math., no. 12, Interscience, New York, 1962. MR 27 \#2808.

15. L. Schwartz, Mathematics for the physical sciences, Hermann, Paris, 1966.

16. G. Szegö, Orthogonal polynomials, Amer. Math. Soc. Colloq. Publ., vol. 23, Amer. Math. Soc., Providence, R.I., 1959. MR 21 \#5029.

17. E. Wagner, Taubersche Sätze reeler Art für die Laplace-Transformation, Math. Nachr. 31 (1966), 153-168. MR 33 \#3003.

18. - Ein reeler Tauberschen Satz für die Laplace-Transformation, Math. Nachr. 36 (1968), 323-331. MR 38 \#1436.

19. D. V. Widder, The Laplace transform, Princeton Math. Series, vol. 6, Princeton Univ. Press, Princeton, N.J., 1941. MR 3, 232.

20. - - Inversion of a heat transform by use of series, J. Analyse Math. 18 (1967), 389-413. MR 35 \#2088.

21. —_, An introduction to transform theory, Pure and Appl. Math., vol. 42, Academic Press, New York, 1971.

22. A. H. Zemanian, Distribution theory and transform analysis. An introduction to generalized functions, with applications, McGraw-Hill, New York, 1965. MR 31 \#1556.

23. V. Riekstyna, Generalized asymptotic expansions for a contour integral, Latvijas Valsts Univ. Zinātn. Raksti 28 (1959), 111-126. MR 23 \#A483.

24. - Asymptotic expansions of some integrals and the sums of power series, Latvian Math. Yearbook 9 (1971), 203-220.

25. E. Wagner, Taubersche Sätze reeler Art für Integraltransformationen mit Kernen der Form $\exp h(s) t$, Wiss. Z. Univ. Rostock 20 (1971), 313-320.

Mathematical Sciences Department, ibM, T. J. Watson Research Center, YORKTOWN HEIGHTS, New YORK 10598 